\title{
Dysnatremia in hospitalized elderly patients
}

\author{
Abeer H. M. Matter \\ Geriatrics and Gerontology department, Faculty of Medicine, Ain Shams University.
}

\begin{abstract}
Background: Dysnatremia is the most common electrolyte disorder in hospitalized patients; it encompasses hyponatremic and hypernatremic conditions. Dysnatremia is also a common finding at Intensive Care Unit admission. Abnormal serum sodium concentrations are known to adversely affect physiologic function and an increasing evidence suggests that dysnatremia may be associated with adverse outcome.
\end{abstract}

Aim: to detect the prevalence of dysnatremia in Geriatric patients, and also to evaluate association between dysnatremia and in-hospital mortality in Intensive Care Unit older patients.

Results: the current study found that in hospitalized elderly participants, $23.8 \%$ had hyponatremia $(6.4 \%, 9.2 \%, 8.2 \%$ had mild, moderate, severe hyponatremia, respectively), $6.8 \%$ had hypernatremia $(2.6 \%, 2 \%, 2.2 \%$ had mild, moderate, severe hypernatremia, respectively) ( $p$ value $=0.000$ ). while prevalence of dysnatremia was higher in the ICU patients, $28.2 \%$ had hyponatremia $(7.6 \%, 9.8 \%, 10.8 \%$ had mild, moderate, severe hyponatremia, respectively), $9.6 \%$ had hypernatremia $(3.3 \%, 3.0 \%, 3.3 \%$ had mild, moderate, severe hypernatremia, respectively) ( $p$ value= 0.000). Intensive Care Unit hospital mortality rate was $40.7 \%$, the dead subjects were older $(p$ value $=0.012$ ), has higher mean s. $\mathrm{Na}$ ( $p$ value=0.002), had higher Modified Acute Physiology and Chronic Health Evaluation II score ( $p$ value $=0.000$ ), higher Multiple Organ Dysfunction score ( $p$ value $=0.000)$, higher length of stay $(p$ value $=0.167)$.

Conclusion: Dysnatremia prevalence is high in geriatric elderly patients, hyponatremia is more frequent than hypernatremia, dysnatremia in Intensive Care Unit patients is more than ward patients, in hospital mortality among geriatric patients with dysnatremia is related to severity and nature of underling diseases as well as severity of dysnatremia itself.

Keywords: dysnatremia; hypernatremia; hyponatremia; geriatric; Intensive Care Unit (ICU).

\section{Background}

Dysnatremia is the most common electrolyte disorder in hospitalized patients; it encompasses hyponatremic and hypernatremic conditions. It is a common finding at Intensive Care Unit (ICU) admission [1-3]. Up to one third of critically ill patients have dysnatremia at ICU admission [2]. Another one-third of critically ill patients will develop an ICU-acquired dysnatremia during ICU stay [4,5]. Prevalence of dysnatremia at ICU admission, however, varies greatly according to the chosen definition $[1,2,6,7]$.

Hyponatremia is a pathologic condition defined as a serum sodium $<135 \mathrm{mmol} / \mathrm{L}$. It is the most common electrolyte disorder in hospitalized patients. Up to $40 \%$ of the overall population of hospitalized patients has a hyponatremia at admission [8]. Presence of severe hyponatremia on hospital admission has been demonstrated to be independently associated with an increased risk for ICU admission and poor prognosis
[9]. Hyponatremia may be due to chronic organ dysfunctions (that is heart failure or liver dysfunction) but also to diuretic use, syndrome of inappropriate antidiuretic hormone (ADH) secretion, adrenal insufficiency, and cerebral or renal salt wasting syndromes $[10,11]$.

Hypernatremia is defined as a serum sodium > 145 $\mathrm{mmol} / \mathrm{L}$. It is generally related to water losses with increase of plasma osmolality. Serum sodium is the main cation of extracellular fluid and plays a key role in serum osmolarity. It is less frequent than hyponatremia [10] with a reported in-hospital prevalence of up to $7.7 \%$ [2]. Since thirst and free access to water are the most important mechanisms that prevent hypernatremia, critically ill patients and older patients are at high risk for this disorder [4,11]. Only $2.5 \%$ patients have been found to develop moderate to severe hypernatremia in the general in-hospital population of patients. Higher prevalence has been 
reported in geriatric or critically ill patients [12-15]. Hypernatremia has multiple adverse effects on physiologic functions, which may explain its association with increased mortality. Hypernatremia aggravates peripheral insulin resistance [16], impairs hepatic gluconeogenesis and lactate clearance $[17,18]$, and decreases left ventricular contractility [19]. Additionally, hypernatremia is associated with various neuromuscular manifestations, such muscle weakness and cramps [20]. Neurologic impairment remains the most severe consequence of hypernatremia and may prolong the need for mechanical ventilation and delay weaning [21]. Finally, too rapid correction of chronic hypernatremia can cause cerebral edema [21].

In addition to critical illness per se, factors contributing to hyponatremia in ICU include excess use of hypotonic fluids and drugs stimulating antidiuretic hormone secretion [22].

\section{Methods}

The present study is a cross-sectional observational study done over 20 months (May 2017 till December 2018) to detect the prevalence of dysnatremia (hyponatremia and hypernatremia) in Geriatric Department (inpatients ward and ICU patients), Ain Shams University Hospitals. Also to evaluate association between dysnatremia and in-hospital mortality in ICU patients. The study was performed in adherence to the principles established with the Declaration of Helsinki and the study methodology was reviewed and approved by the Research Review Board of the Geriatric and Gerontology Department, Faculty of Medicine, Ain Shams University.

Selection criteria:

1. Age of 60 years or older.

2. Verbal consent obtained from conscious patients.

3. Informed consent taken from surrogate of unconscious cases.

\section{Exclusion criteria}

1. Patients who are less than 60 years' old

2. Patients who refuse to participate in the research

3. Patients with hyperlipidemia, paraproteinemias (known cases), as well as those receiving mannitol were excluded from the study.

4. Patients stay less than 48 hours at ICU.

Study tool: All patients were evaluated by history and clinical examination.

Serum sodium (s. Na) level was measured for all participants at hospital admission and followed during hospital admission.

Based on s. Na level patients were classified into mild (130-134 mg/dl), moderate (125-129 mg/dl) and severe hyponatremia (<125 mg/dl), normal subjects (135-145 $\mathrm{mg} / \mathrm{dl})$, mild hypernatremia (146-149 mg/dl), moderate hypernatremia (150-159 mg/dl), severe hypernatremia $(\geq 160 \mathrm{mg} / \mathrm{dl})$.

For ICU patient's clinical data (cause of admission, Modified Acute Physiology and Chronic Health Evaluation (APACHE) II score [23], Multiple Organ Dysfunction (MOD) score [24]), length of ICU stays in days, in hospital mortality, cause of death) were detected.

Statistical analysis: The statistical analysis of the research was performed with the SPSS 22.0 package program. Descriptive statistics were carried out for all variables and expressed as mean and \pm SD for quantitative data, whereas qualitative data were expressed as numbers and percentages.

Comparisons between means were made with the Student's t test. Distributions were compared with the Chi-square test. Data are expressed as means with standard deviations. Logistic regression analysis was used to detect factors affecting in hospital mortality. A $\mathrm{p}$ value $<0.05$ was considered statistically significant.

\section{Results}

502 elderlies were recruited in the study (305 icu+197 inpatient ward) with mean age 70.72 $\pm 8.67,42.4 \%$ male and $57.6 \%$ female, Mean s. Na $137.33 \pm 8.29$, $30.48 \%$ had dysnatremia, $6.6 \%$ (33/502) had symptomatic dysnatremia, more than half of the participants died in hospital $(56.4 \%)$, half of the participants had diabetes Mellitus (50.2\%), and hypertension $(58.6 \%), 20.9 \%$ had cardiac problems, $29.5 \%$ had renal impairment, $19.9 \%$ had liver cell failure.

Of all participants, $23.8 \%$ had hyponatremia $(6.4 \%, 9.2 \%, 8.2 \%$ had mild, moderate, severe hyponatremia, respectively), $6.8 \%$ had hypernatremia $(2.6 \%, 2 \%, 2.2 \%$ had mild, moderate, severe hypernatremia, respectively) ( $\mathrm{p}$ value $=0.000$ ).

Hyponatremic patients were younger (mean age $68.16 \pm 7.54)$, with female predominance, most of them (69/119) had HTN (57.98\%), 51.3\% had DM (61/119), $39.5 \%$ had CKD, $18.5 \%$ (22/119) had symptomatic hyponatremia, $25.21 \%(30 / 119)$ of hyponatremic patients died in hospital.

Hypernatremic subjects were older (mean age $73.62 \pm 9.19)$ with female predominance, most of them 21 participants had HTN $(61.76 \%), 16$ participants had HTN and CKD (37.21\%), 11 had symptomatic hypernatremia $(32.35 \%)$, with higher mortality rate $61.76 \%(21 / 34)$.

There are a high significant association between grades of $\mathrm{s}$. $\mathrm{Na}$ ( $\mathrm{p}$ value=0.000), also a high significant association present in between groups as regard age, sex, mean s. Na, symptoms, in hospital mortality, presence of renal impairment and liver cell failure. 
Table 1: Patients' demographic, clinical, and outcome characteristics, association of dysnatremia with these factors.

\begin{tabular}{|c|c|c|c|c|c|c|c|c|c|c|c|}
\hline & \multirow[t]{2}{*}{ Total } & \multicolumn{4}{|c|}{ Hyponatremia } & \multirow{2}{*}{$\begin{array}{l}\text { Normal } \\
\text { Normal }\end{array}$} & \multicolumn{4}{|c|}{ Hypernatremia } & \multirow[t]{2}{*}{ Sig. } \\
\hline & & All & Severe & $\begin{array}{l}\text { Mode } \\
\text { rate }\end{array}$ & Mild & & All & Mild & $\begin{array}{l}\text { Moder } \\
\text { ate }\end{array}$ & Severe & \\
\hline $\begin{array}{l}\text { No } \\
(\%)\end{array}$ & $\begin{array}{l}502 \\
(100)\end{array}$ & $\begin{array}{l}119 \\
(23.7)\end{array}$ & $\begin{array}{l}41 \\
(8.2)\end{array}$ & $\begin{array}{l}46 \\
(9.2)\end{array}$ & $\begin{array}{l}32 \\
(6.4)\end{array}$ & $\begin{array}{l}349 \\
(69.5)\end{array}$ & $\begin{array}{l}34 \\
(6.77)\end{array}$ & $\begin{array}{l}13 \\
(2.6)\end{array}$ & $\begin{array}{l}10 \\
(2)\end{array}$ & $\begin{array}{l}11 \\
(2.2)\end{array}$ & 0.000 \\
\hline $\begin{array}{l}\text { Age } \\
\text { mean } \pm \\
\text { SD }\end{array}$ & $\begin{array}{l}70.72 \pm \\
8.67\end{array}$ & $\begin{array}{l}68.16 \pm 7 \\
.54\end{array}$ & $\begin{array}{l}69.61 \pm \\
7.44\end{array}$ & $\begin{array}{l}67.07 \\
\pm 6.96\end{array}$ & $\begin{array}{l}67.87 \pm \\
8.37\end{array}$ & $\begin{array}{l}71.35 \pm 8 \\
.75\end{array}$ & $\begin{array}{l}73.62 \pm \\
9.19\end{array}$ & $\begin{array}{l}76.00 \\
\pm 10.84\end{array}$ & $\begin{array}{l}73.10 \\
\pm 9.35\end{array}$ & $\begin{array}{l}71.27 \pm \\
6.75\end{array}$ & 0.003 \\
\hline $\begin{array}{l}\text { s. } \mathrm{Na} \\
\text { mean } \pm \\
\text { SD }\end{array}$ & $\begin{array}{l}137.33 \\
\pm 8.30\end{array}$ & $\begin{array}{l}126.45 \pm \\
5.35\end{array}$ & $\begin{array}{l}120.46 \\
\pm 4.28\end{array}$ & $\begin{array}{l}127.9 \\
8 \pm 1.2 \\
7\end{array}$ & $\begin{array}{l}131.94 \\
\pm 0.91\end{array}$ & $\begin{array}{l}139.14 \pm \\
2.86\end{array}$ & $\begin{array}{l}155.35 \\
\pm 7.70\end{array}$ & $\begin{array}{l}148.23 \\
\pm 1.24\end{array}$ & $\begin{array}{l}154.20 \\
\pm 2.53\end{array}$ & $\begin{array}{l}164.82 \\
\pm 4.71\end{array}$ & 0.000 \\
\hline $\begin{array}{l}\text { Male } \\
(\%)\end{array}$ & $\begin{array}{l}213 \\
(42.4)\end{array}$ & $\begin{array}{l}42 \\
(8.37)\end{array}$ & $\begin{array}{l}11 \\
(2.19)\end{array}$ & $\begin{array}{l}18 \\
(3.59)\end{array}$ & $\begin{array}{l}13 \\
(2.59)\end{array}$ & $\begin{array}{l}157 \\
(28.88)\end{array}$ & $\begin{array}{l}14 \\
(2.79)\end{array}$ & $\begin{array}{l}5 \\
(1)\end{array}$ & $\begin{array}{l}4 \\
(0.8)\end{array}$ & $\begin{array}{l}5 \\
(1)\end{array}$ & 0.492 \\
\hline $\begin{array}{l}\text { DM } \\
(\%)\end{array}$ & $\begin{array}{l}252 \\
(50.2)\end{array}$ & $\begin{array}{l}61 \\
(12.15)\end{array}$ & $\begin{array}{l}20 \\
(3.98)\end{array}$ & $\begin{array}{l}26 \\
(5.18)\end{array}$ & $\begin{array}{l}15 \\
(2.99)\end{array}$ & $\begin{array}{l}175 \\
(34.86)\end{array}$ & $\begin{array}{l}16 \\
(3.19)\end{array}$ & $\begin{array}{l}5 \\
(1)\end{array}$ & $\begin{array}{l}6 \\
(1.2)\end{array}$ & $\begin{array}{l}5 \\
(1)\end{array}$ & 0.838 \\
\hline $\begin{array}{l}\text { HTN } \\
(\%)\end{array}$ & $\begin{array}{l}294 \\
(58.6)\end{array}$ & $\begin{array}{l}69 \\
(13.75)\end{array}$ & $\begin{array}{l}28 \\
(5.58)\end{array}$ & $\begin{array}{l}25 \\
(4.98)\end{array}$ & $\begin{array}{l}16 \\
(3.19)\end{array}$ & $\begin{array}{l}204 \\
(40.64)\end{array}$ & $\begin{array}{l}21 \\
(4.18)\end{array}$ & $\begin{array}{l}6 \\
(1.2)\end{array}$ & $\begin{array}{l}7 \\
(1.39)\end{array}$ & $\begin{array}{l}8 \\
(1.59)\end{array}$ & 0.521 \\
\hline $\begin{array}{l}\text { Card. } \\
(\%)\end{array}$ & $\begin{array}{l}105 \\
(20.9)\end{array}$ & $\begin{array}{l}33 \\
(6.57)\end{array}$ & $\begin{array}{l}14 \\
(2.79)\end{array}$ & $\begin{array}{l}13 \\
(2.59)\end{array}$ & $\begin{array}{l}6 \\
(1.2)\end{array}$ & $\begin{array}{l}65 \\
(12.9)\end{array}$ & $\begin{array}{l}7 \\
(1.4)\end{array}$ & $\begin{array}{l}5 \\
(1)\end{array}$ & $\begin{array}{l}1 \\
(0.2)\end{array}$ & $\begin{array}{l}1 \\
(0.2)\end{array}$ & 0.187 \\
\hline
\end{tabular}

305 elderly patient recruited from ICU with mean age $70.46 \pm 8.65$, mean s. Na $136.99 \pm 9.47,40.7 \%$ were males, $52.5 \%$ had pulmonary problems, $54.1 \%$ had DM, $59.7 \%$ had HTN, $18.4 \%$ had cardiac problems, $39.7 \%$ had renal impairment, $21.0 \%$ had liver cell failure, with mean Modified Acute Physiology and Chronic Health Evaluation II (modified APACHE II) score is $9.90 \pm 5.41$, $(\mathrm{p}$ value $=0.000)$ mean MOD (Multiple Organ Dysfunction) score is $6.78 \pm 4.9$ (p value $=0.000)$, mean length of ICU stay is $7.15 \pm 7.49$ (p value $=0.006), 10.2 \%$ had symptomatic dysnatremia $(p$ value $=0.000), 31.5 \%$ had respiratory problems as a cause of admission (most common cause), followed by shock $16.4 \%$, followed by cardiac causes $13.4 \%$ ( $p$ value $=0.000)$.

In the ICU patients, $28.2 \%$ had hyponatremia (7.6\%, $9.8 \%, 10.8 \%$ had mild, moderate, severe hyponatremia, respectively), $9.6 \%$ had hypernatremia $(3.3 \%, 3.0 \%$, $3.3 \%$ had mild, moderate, severe hypernatremia, respectively) $(\mathrm{p}$ value $=0.000)$.
In ICU hospital mortality was $40.7 \%$ ( $\mathrm{p}$ value=0.001), mortality in hyponatremic, normonatremic, hypernatremic patients were $33.7 \%, 38.9 \%, 72.4 \%$, respectively. Most common cause of death in hyponatremic and normonatremic patients was respiratory, followed by metabolic causes, then cardiac and other causes, while most common cause of death in hypernatremic patients was metabolic causes followed by respiratory failure then cardiac and other causes ( $p$ value $=0.000)$.

In hospital mortality rate in mild, moderate, and severe hyponatremia was $(47.8 \%, 16.7 \%, \quad 39.4 \%$, respectively), while in hospital mortality rate in mild, moderate, and severe hypernatremia was $(60 \%, 100 \%$, $60 \%)$.Hospital acquired hyponatremia incidence was $2.6 \%(0.6 \%, 1.2 \%, 0.8 \%$ for mild, moderate, severe hyponatremia, respectively),

Hospital acquired hypernatremia incidence was $3.6 \%$ $(1.8 \%, 1 \%, 0.8 \%$ for mild, moderate, severe hypernatremia, respectively. 
Matter AHM., EJGG.2019; 6(2):38-44

Table (2): ICU Patients' demographic, clinical, and outcome characteristics, association of dysnatremia with these factors.

\begin{tabular}{|c|c|c|c|c|c|c|c|c|c|c|c|}
\hline & \multirow[t]{2}{*}{ Total } & \multicolumn{4}{|l|}{ Hyponatremia } & \multirow{2}{*}{$\begin{array}{l}\text { Normal } \\
\text { Normal }\end{array}$} & \multicolumn{5}{|c|}{ Hypernatremia } \\
\hline & & All & Severe & Moderate & Mild & & All & Mild & Moderate & Severe & \\
\hline No & 305 & 86 & 33 & 30 & 23 & 190 & 29 & 10 & 9 & 10 & 0.000 \\
\hline$(\%)$ & $100 \%$ & $28.2 \%$ & $10.8 \%$ & $9.8 \%$ & $7.6 \%$ & $62.3 \%$ & $9.51 \%$ & $3.28 \%$ & $2.95 \%$ & $3.28 \%$ & \\
\hline $\begin{array}{l}\text { Age } \\
\text { mean } \pm S D\end{array}$ & $\begin{array}{l}70.46 \pm \\
8.65\end{array}$ & $68.06 \pm 7.20$ & $68.48 \pm 6.88$ & $68.30 \pm 7.23$ & $67.13 \pm 7.81$ & $71.32 \pm 8.74$ & $73.59 \pm 9.10$ & $76.20 \pm 10.43$ & $73.67 \pm 9.73$ & $\begin{array}{l}70.90 \pm 6 \\
99\end{array}$ & 0.010 \\
\hline $\begin{array}{l}\text { s. } \mathrm{Na} \\
\text { mean } \pm \mathrm{SD}\end{array}$ & $136.99 \pm 9.48$ & $125.98 \pm 5.43$ & $120.58 \pm 4.58$ & $127.57 \pm 1.10$ & $131.61 \pm 0.99$ & $139.08 \pm 2.85$ & $155.90 \pm 7.66$ & $148.40 \pm 1.27$ & $154.44 \pm 2.56$ & $\begin{array}{l}164.70 \pm 4 \\
.95\end{array}$ & 0.000 \\
\hline \multirow[t]{2}{*}{ Male (\%) } & 124 & 29 & 9 & 11 & 9 & 82 & 13 & 4 & 4 & 5 & 0.733 \\
\hline & $40.7 \%$ & $9.5 \%$ & $2.95 \%$ & $3.6 \%$ & $2.95 \%$ & $26.89 \%$ & $4.26 \%$ & $1.3 \%$ & $1.3 \%$ & $1.64 \%$ & \\
\hline DM & 165 & 51 & 20 & 18 & 13 & 99 & 15 & 4 & 6 & 5 & 0.840 \\
\hline (\%) & $54.1 \%$ & $59.3 \%$ & $60.6 \%$ & $60.0 \%$ & $56.5 \%$ & $52.1 \%$ & $51.7 \%$ & $40 \%$ & $66.7 \%$ & $50 \%$ & \\
\hline HTN & 182 & 51 & 22 & 17 & 12 & 113 & 18 & 4 & 7 & 7 & 0.840 \\
\hline (\%) & $59.7 \%$ & $59.3 \%$ & $66.7 \%$ & $56.7 \%$ & $52.2 \%$ & $59.5 \%$ & $62.1 \%$ & $40 \%$ & $77.8 \%$ & $70 \%$ & \\
\hline Pulm. & 160 & 33 & 11 & 12 & 10 & 104 & 22 & 8 & 6 & 8 & 0.028 \\
\hline (\%) & $52.5 \%$ & $38.4 \%$ & $33.3 \%$ & $40.0 \%$ & $43.5 \%$ & $54.7 \%$ & $75.9 \%$ & $80 \%$ & $66.7 \%$ & $80 \%$ & \\
\hline Card. & 56 & 23 & 12 & 8 & 3 & 26 & 7 & 4 & 1 & 2 & 0.018 \\
\hline (\%) & $18.4 \%$ & $26.7 \%$ & $36.4 \%$ & $26.7 \%$ & $13.0 \%$ & $13.7 \%$ & $24.1 \%$ & $40 \%$ & $11.1 \%$ & $20 \%$ & \\
\hline CKD & 121 & 42 & 16 & 17 & 9 & 64 & 15 & 3 & 5 & 7 & 0.047 \\
\hline (\%) & $39.7 \%$ & 48.8 & $48.5 \%$ & $56.7 \%$ & $2.95 \%$ & $33.7 \%$ & $51.7 \%$ & $30 \%$ & $55.6 \%$ & $70 \%$ & \\
\hline LCF & 64 & 26 & 11 & 7 & 8 & 37 & 1 & 1 & 0 & 0 & 0.065 \\
\hline (\%) & $21.0 \%$ & 30.2 & $33.3 \%$ & $23.3 \%$ & $34.8 \%$ & $19.5 \%$ & $3.4 \%$ & $10 \%$ & $0 \%$ & $0 \%$ & \\
\hline Symp & 31 & 21 & 15 & 6 & 0 & 0 & 10 & 0 & 4 & 6 & 0.000 \\
\hline (\%) & $10.2 \%$ & $24.4 \%$ & $45.5 \%$ & $20.0 \%$ & $0 \%$ & $0 \%$ & $34.5 \%$ & $0 \%$ & $44.4 \%$ & $60 \%$ & \\
\hline Primary $c$. & & & & & & & & & & & 0.000 \\
\hline $\mathrm{RF}$ & 96 & 22 & 8 & 8 & 6 & 70 & 4 & 3 & 0 & 1 & \\
\hline (\%) & $31.5 \%$ & $25.6 \%$ & $24.2 \%$ & $26.7 \%$ & $26.1 \%$ & $36.8 \%$ & $13.8 \%$ & $30 \%$ & $0 \%$ & $10 \%$ & \\
\hline Cardiac & 41 & 13 & 6 & 7 & 0 & 27 & 1 & 1 & 0 & 0 & \\
\hline (\%) & $13.4 \%$ & $15.1 \%$ & $18.2 \%$ & $23.3 \%$ & $0 \%$ & $14.2 \%$ & $3.4 \%$ & $10 \%$ & $0 \%$ & $0 \%$ & \\
\hline Shock & 50 & 10 & 2 & 3 & 5 & 33 & 7 & 3 & 3 & 1 & \\
\hline (\%) & $16.4 \%$ & $11.6 \%$ & $6.1 \%$ & $10.0 \%$ & $21.7 \%$ & $17.4 \%$ & $24.1 \%$ & $30 \%$ & $33.3 \%$ & $10 \%$ & \\
\hline \multirow[t]{2}{*}{ metabolic } & 35 & 15 & 7 & 5 & 3 & 15 & 5 & 1 & 0 & 4 & \\
\hline & $11.5 \%$ & $17.4 \%$ & $21.2 \%$ & $16.7 \%$ & $13.0 \%$ & $7.9 \%$ & $17.2 \%$ & $10 \%$ & $0 \%$ & $40 \%$ & \\
\hline Neuro. & 34 & 5 & 1 & 2 & 2 & 19 & 10 & 1 & 5 & 4 & \\
\hline (\%) & $11.1 \%$ & $5.8 \%$ & $3.0 \%$ & $6.7 \%$ & $8.7 \%$ & $10.0 \%$ & $34.5 \%$ & $10 \%$ & $55.6 \%$ & $40 \%$ & \\
\hline LCF & 21 & 12 & 5 & 2 & 5 & 9 & 0 & 0 & 0 & 0 & \\
\hline (\%) & $6.9 \%$ & $14.0 \%$ & $15.2 \%$ & $6.7 \%$ & $21.7 \%$ & $2.95 \%$ & $0 \%$ & $0 \%$ & $0 \%$ & $0 \%$ & \\
\hline Others. & 28 & 9 & 4 & 3 & 2 & 17 & 2 & 1 & 1 & 0 & \\
\hline (\%) & $9.18 \%$ & $2.95 \%$ & $12.12 \%$ & $10 \%$ & $8.7 \%$ & $8.95 \%$ & $6.9 \%$ & $10 \%$ & $11.1 \%$ & $0 \%$ & \\
\hline
\end{tabular}

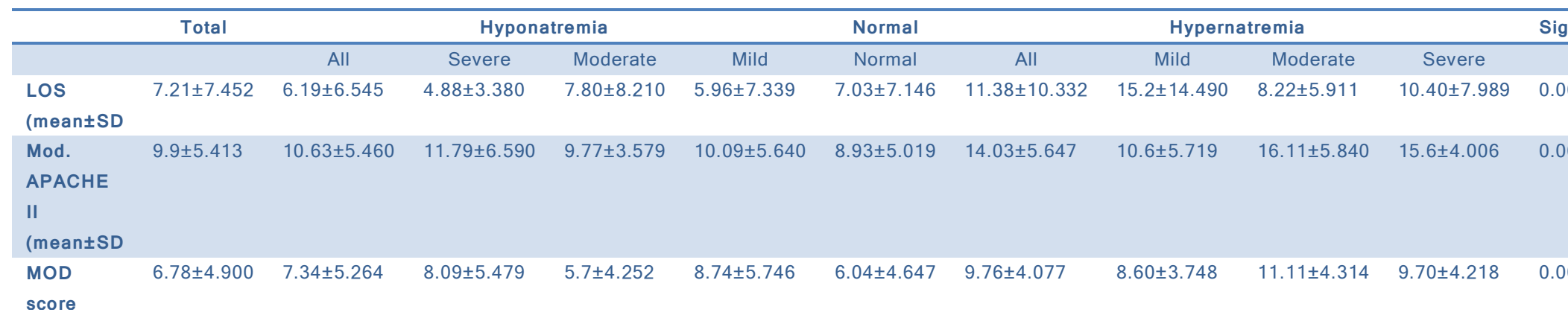


Table (3): comparison between survived and in hospital mortality groups.

\begin{tabular}{|c|c|c|c|}
\hline & $\begin{array}{c}\text { Survived } \\
181(59.3 \%)\end{array}$ & $\begin{array}{c}\text { In hospital mortality } \\
124(40.7 \%)\end{array}$ & Sig. \\
\hline Age (mean $\pm S D)$ & $69.60 \pm 8.237$ & $72.09 \pm 8.739$ & 0.012 \\
\hline s. $\mathrm{Na}($ meantSD) & $135.62 \pm 8.244$ & $138.98 \pm 10.765$ & 0.002 \\
\hline DM (\%) & $101(55.8 \%)$ & $64(51.6 \%)$ & 0.471 \\
\hline HTN(\%) & $106(58.6 \%)$ & $76(61.3 \%)$ & 0.633 \\
\hline Cardiac(\%) & $28(15.5 \%)$ & $28(22.6 \%)$ & 0.115 \\
\hline pulmonary(\%) & $72(39.8 \%)$ & $88(71 \%)$ & 0.000 \\
\hline CKD(\%) & $69(38.1 \%)$ & $52(41.9 \%)$ & 0.504 \\
\hline $\operatorname{LCF}(\%)$ & $40(22.1 \%)$ & $24(19.4 \%)$ & 0.563 \\
\hline $\begin{array}{l}\text { Symptomatic } \\
\text { (\% of total) }\end{array}$ & $15(8.3 \%)$ & $16(12.9 \%)$ & 0.190 \\
\hline MOD score & $4.29 \pm 3.204$ & $10.42 \pm 4.677$ & 0.000 \\
\hline length of stay & $6.72 \pm 6.776$ & $7.92 \pm 8.318$ & 0.167 \\
\hline
\end{tabular}

Table (4): Factors associated with in hospital mortality (logistic regression analysis)

\begin{tabular}{cccc}
\hline & Odds Ratio & S5\% CI & \\
\hline Age & 1.109 & $(0.747-1.647)$ & \\
s. Na & 1.194 & $(1.054-1.353)$ & 0.609 \\
Mod. APACHEII score & 1.305 & $(1.186-1.436)$ & 0.005 \\
MOD score & 1.275 & $(1.164-1.397)$ & 0.000 \\
Length of stay & 1.029 & $(0.984-1.076)$ & 0.000 \\
\hline
\end{tabular}

\section{Discussion:}

The current study done to assess prevalence of dysnatremia in hospitalized geriatric population and to study the association of dysnatremia in elderly with in hospital Intensive Care Unit (ICU) mortality, the current study found that in hospitalized elderly participants, $23.8 \%$ had hyponatremia $(6.4 \%, 9.2 \%$, $8.2 \%$ had mild, moderate, severe hyponatremia, respectively), $6.8 \%$ had hypernatremia $(2.6 \%, 2 \%$, $2.2 \%$ had mild, moderate, severe hypernatremia, respectively) ( $p$ value $=0.000)$. while prevalence of dysnatremia was higher in the ICU patients, $28.2 \%$ had hyponatremia $(7.6 \%, 9.8 \%, 10.8 \%$ had mild, moderate, severe hyponatremia, respectively), $9.6 \%$ had hypernatremia $(3.3 \%, 3.0 \%, 3.3 \%$ had mild, moderate, severe hypernatremia, respectively) ( $\mathrm{p}$ value $=0.000$ ).

Earlier studies reported high prevalence of dysnatremia in hospitalized ICU patients, as in Darmon et al. 2013[25] study who found that one-third of critically ill patients had a moderate to severe dysnatremia at ICU, Mild to severe hypernatremia and hyponatremia were present in respectively $7.8 \%$ and $27.4 \%$ of the admitted patients, $18.02 \%$ had mild hyponatremia, $6.23 \%$ had moderate hyponatremia, $3.14 \%$ had severe hyponatremia, $5.69 \%$ had mild hypernatremia, $1.29 \%$ had moderate hypernatremia, and $0.91 \%$ had severe hypernatremia. In Funk et al. 2010 [2] study found that the frequencies of borderline (130-135 mmol/L), mild (125-130 $\mathrm{mmol} / \mathrm{L})$, and severe hyponatremia (s. Na < $125 \mathrm{mmol} / \mathrm{L})$ were $13.8 \%, 2.7 \%$, and $1.2 \%$, respectively. The frequencies of borderline (145-150 $\mathrm{mmol} / \mathrm{L})$, mild $(150-155 \mathrm{mmol} / \mathrm{L})$, and severe hypernatremia (s. $\mathrm{Na}>155 \mathrm{mmol} / \mathrm{L}$ ) were $5.1 \%, 1.2 \%$, and $0.6 \%$, respectively. Bennani et al.2003 [1] found the prevalence of hyponatremia, (s. Na $<130 \mathrm{mmol} / \mathrm{L}$ ) on admission to the ICU to be $14 \%$ compared with our finding of about $20.6 \%$ (moderate and severe hyponatremia, s. Na <130).

In our study, ICU hospital mortality rate was $40.7 \%$, the died subjects were older ( $p$ value $=0.012)$, has higher mean $s$. $\mathrm{Na}$ ( $\mathrm{p}$ value $=0.002$ ), had higher Modified Acute Physiology and Chronic Health Evaluation II (modified APACHE II) score (p value $=0.000)$, higher Multiple Organ Dysfunction (MOD) score ( $p$ value $=0.000)$, higher length of stay $(p$ value $=0.167)$.

In hospital mortality in moderate hypernatremia more than mild and severe hypernatremia as that group (moderate hypernatremia) had the worst modified 
APACHE II score and MOD score. Also this illustrate why in hospital mortality in mild hyponatremia was more than moderate and severe hyponatremic groups, so, the nature, severity of underlying illness as well as the severity of dysnatremia best explain in hospital mortality associated with dysnatremia in geriatric ICU population. Several lines of evidence from our analysis support this conclusion: (1) once s. Na falls below 130 $\mathrm{mEq} / \mathrm{L}$ (moderate hyponatremia), or rises above 160 $\mathrm{mEq} / \mathrm{L}$ (severe hypernatremia), mortality rate does not seem to increase as the severity of hyponatremia / hypernatremia worsens; in fact, the trend is in the opposite direction. (2) in hospital mortality in patients with s. $\mathrm{Na}$ (150-159) $\mathrm{mEq} / \mathrm{L}$ were associated with serious comorbidities as illustrated by worst modified APACHE II score and worst MOD score and this ensures that in hospital mortality in geriatric population associated with severity of underling diseases not with dysnatremia alone, this agreed with Chawla et al., 2011, [26] who found that mortality rate tended to increase as the s. Na fell from 134 to $120 \mathrm{mEq} / \mathrm{L}$ but as the s. Na fell below $120 \mathrm{mEq} / \mathrm{L}$, the trend reversed, so that mortality among the patients with $\mathrm{s}$. Na $<115$ $\mathrm{mEq} / \mathrm{L}(6.8 \%)$ was considerably lower than the mortality among the 1844 patients in the 120 to 124 $\mathrm{mEq} / \mathrm{L}(11.2 \%)$. All patients who died had significant acute progressive underlying illnesses. A paradoxical fall in mortality rate as the $\mathrm{s}$. $\mathrm{Na}$ falls can be found in other studies as well [27-29].

An increase in the risk of in-hospital mortality in patients with dysnatremia has been reported in several studies, Darmon et al. 2013[25] study found that, mortality at day 30 was $21.0 \%$ in the overall population of patients, Day-30 mortality was of $16.6 \%$ in patients without dysnatremia at ICU admission, $19.0 \%$ in patients with borderline hyponatremia, $22.2 \%$ in patients with mild hyponatremia, $26.4 \%$ in patients with moderate hyponatremia and $23.8 \%$ in patients with severe hyponatremia. Day-30 mortality was $22.5 \%$ in patients with borderline hypernatremia, $32.1 \%$ in patients with mild hypernatremia, $39.9 \%$ in patients with moderate hypernatremia and $54.5 \%$ in patients with severe hypernatremia. Bennani et al.2003 [1] showed that severe hyponatremia ( $\mathrm{s}$. Na $<125 \mathrm{mmol} / \mathrm{L}$ ) on ICU admission was independently associated with an increase in mortality. In Funk et al. 2010 [2] concluded that all types and grades of dysnatremia were associated with increased raw and risk-adjusted hospital mortality ratios.

In our study, incidence of ICU Hospital acquired (HA) hypernatremia is more than ICU hospital acquired (HA) hyponatremia $(3.6 \%$ versus $2.6 \%)$, among HA hyponatremia $0.6 \%, 1.2 \%, 0.8 \%$ had mild, moderate, severe hyponatremia, respectively, among hospital acquired hypernatremia $1.8 \%, 1 \%, 0.8 \%$ had mild, moderate, severe hypernatremia, respectively.

The incidence of in-hospital hyponatremia and hypernatremia has been described ranging from 9.0 to $13.6 \%$ and from 1.0 to $9.1 \%$ [30,31], respectively, also
Lombardi et al 2019 [32] found that HA hyponatremia and hypernatremia was 10.7 and $4.3 \%$, respectively. Stelfox et al., 2008 [7] found that hospital acquired hyponatremia developed in 917 (11\%) patients and hypernatremia in $2157(26 \%)$ patients.

Explanations for differences in dysnatremia epidemiology compared with previous works include study design (retrospective-prospective), sample sizes, differences in study population, age groups and in cutoff levels used to define dysnatremia.

Conclusion:

Dysnatremia prevalence is high in geriatric elderly patients, hyponatremia is more common than hypernatremia, dysnatremia in ICU patients more than ward patients, in hospital mortality in geriatric patients having dysnatremia is most probably due to severity, nature of underling diseases as well as severity of dysnatremia, according to regression analysis, s. $\mathrm{Na}$ is also a predictor of mortality with disease severity.

\section{References}

1] Bennani, S.L., Abouqal, R., Zeggwagh, A.A., Madani, N., Abidi, K., Zekraoui, A. and Kerkeb, O. (2003) Incidence, causes and prognostic factors of hyponatremia in intensive care. Rev Med Interne, 24, 224-229.

2] Funk, G.C., Lindner, G., Druml, W., Metnitz, B., Schwarz, C., and Bauer, $P$. et al. (2010) Incidence and prognosis of dysnatremias present on ICU admission. Intensive Care Med. 36(2), 304-11.

3] Lindner, G., Funk, G.C., Schwarz, C., Kneidinger, N., Kaider, A., Schneeweiss, B., Kramer, L. and Druml, W. (2007) Hypernatremia in the critically ill is an independent risk factor for mortality. Am J Kidney Dis. 50, 952-957.

4] Hoorn, E.J., Betjes, M.G.H., Weigel, J., and Zietse, R. (2008) Hypernatraemia in critically ill patients: too little water and too much salt. Nephrol Dial Transplant, 23, 1562-1568.

5] Darmon, M., Timsit, J.F., Francais, A., Nguile-Makao, M., Adrie, C., Cohen, Y., Garrouste-Orgeas, M., Goldgran-Toledano, D., Dumenil, A-S., Jamali, S., Cheval, C., Allaouchiche, B., Souweine, B., and Azoulay, E. (2010) Association between hypernatraemia acquired in the ICU and mortality: a cohort study. Nephrol Dial Transplant, 25, 2510-2515.

6] Stelfox, H.T., Ahmed, S.B., Zygun, D., Khandwala, F., and Laupland, K. (2010) Characterization of intensive care unit acquired hyponatremia and hypernatremia following cardiac surgery. Can J Anaesth, 57, 650-658.

7] Stelfox, H.T., Ahmed, S.B., Khandwala, F., Zygun, D., Shahpori, R., and Laupland, K. (2008) The epidemiology of intensive care unit-acquired hyponatraemia and hypernatraemia in medical-surgical intensive care units. Crit Care. 12(6), R162.

8] Sedlacek, M., Schoolwerth, A.C., and Remillard, B.D. (2006) Electrolyte disturbances in the intensive care unit. Semin Dial, 19, 496-501.

9] Zilberberg, M.D., Exuzides, A., Spalding, J., Foreman, A., Jones, A.G., Colby, C., and Shorr, A.F. (2008) Epidemiology, clinical and economic outcomes of admission hyponatremia among hospitalized patients. Curr Med Res Opin, 24,1601-1608.

10] Kumar, S. and Berl, T. (1998) Sodium. Lancet., 352(9123), 220-8.

11] Adrogué, H.J., and Madias, N.E. (2000) Hyponatremia. N Engl J Med, 342, 1581-1589.

12] Macdonald, N.J., McConnell, K.N., Stephen, M.R., and Dunnigan, M.G. (1989) Hypernatraemic dehydration in patients in a large hospital for the mentally handicapped. BMJ, 299, 1426-1429.

13] Palevsky, P.M., Bhagrath, R., and Greenberg, A. (1996) Hypernatremia in hospitalized patients. Ann Intern Med, 124, 197-203.

14] Long, C.A., Marin, P., Bayer, A.J., Shetty, H.G., and Pathy, M.S. (1991) Hypernatraemia in an adult in-patient population. Postgrad Med J, 67, 643645 .

15] Borra, S.I., Beredo, R., and Kleinfeld, M. (1995) Hypernatremia in the aging: causes, manifestations, and outcome. J Natl Med Assoc, 87,220224.

16] Bratusch-Marrain, P.R., and DeFronzo, R.A. (1983) Impairment of insulin-mediated glucose metabolism by hyperosmolality in man. Diabetes, 32,1028-1034. 
Matter AHM., EJGG.2019; 6(2):38-44

17] Lenz, K., Gossinger, H., Laggner, A., Druml, W., Grimm, G., and Schneeweiss, B. (1986) Influence of hypernatremic hyperosmolar state on hemodynamics of patients with normal and depressed myocardial function. Crit Care Med, 14, 913-914.

18] Druml, W., Kleinberger, G., Lenz, K., Laggner, A., Schneeweiss, B. (1986) Fructose-induced hyperlactemia in hyperosmolar syndromes. Klin Wochenschr, 64, 615-618.

19] Kozeny, G.A., Murdock, D.K., Euler, D.E., Hano, J.E., Scanlon, P.J., Bansal, V.K., and Vertuno, L.L. (1985) In vivo effects of acute changes in osmolality and sodium concentration on myocardial contractility. Am Heart J, 109, 290-296.

20] Knochel, J.P. (1982) Neuromuscular manifestations of electrolyte disorders. Am J Med, 72,521-535.

21] Adrogue, H.J., and Madias, N.E. (2000) Hypernatremia. N Engl J Med, 342,1493-1499.

22] Hoorn, E.J., Lindemans, J., and Zietse, R. (2006) Development of severe hyponatraemia in hospitalized patients: treatment-related risk factors and inadequate management. Nephrol Dial Transplant., 21, 70-6.

23] Knaus, W.A., Draper, E.A., Wagner, D.P., and Zimmerman, J.E. (1985) APACHE II: a severity of disease classification system. Crit Care Med, 13, 818-29.

24] Marshall, J.C., Cook, D.J., and Christou, N.V., et. al. (1995) Multiple organ dysfunction score: a reliable descriptor of a complex clinical outcome. Crit Care Med., 23(10), 1638-52. Review.

25] Darmon, M., Diconne, E., Souweine, B., Ruckly, S., Adrie, C., Azoulay, E., Clec'h, C., Garrouste-Orgeas, M., Schwebel, C., Goldgran-Toledano, D., Khallel, H., Dumenil, A., Jamali, S., Cheval, C., Allaouchiche, B., Zeni F., And Timsit J.F. (2013) Prognostic consequences of borderline dysnatremia: pay attention to minimal serum sodium change. Critical Care, $17, R 12$.

26] Chawla, A., Sterns, R.H., Nigwekar, S.U. and Cappuccio J.D. (2011).Clin J Am Soc Nephrol, 6, 960 -965.

27] Waikar, S.S., Mount, D.B., and Curhan, G.C. (2009) Mortality after hospitalization with mild, moderate, and severe hyponatremia. Am J Med, 122, 857- 865 .

28] Baran, D., and Hutchinson, T.A. (1984) The outcome of hyponatremia in a general hospital population. Clin Nephrol, 22, 72-76.

29] Clayton, J.A., Le Jeune, I.R., and Hall, I.P. (2006) Severe hyponatraemia in medical in-patients: Aetiology, assessment and outcome. QJM, 99, 505511.

30] Hu, J., Wang, Y., Geng, X., Chen, R., Zhang, P., and Lin, J., et al. (2017) Dysnatremia is an Independent Indicator of Mortality in Hospitalized Patients. Med Sci Monit., 23, 2408-25.

31] Sakr, Y., Rother, S., Ferreira, A.M., Ewald, C., Dünisch, P., and Riedemmann, N., et al. (2013) Fluctuations in serum sodium level are associated with an increased risk of death in surgical ICU patients. Crit Care Med., 41(1), 133-42.

32] Lombardi, G., Naticchia, A., Ferraro, P.M., Calvaruso, L., D'Alonzo, S., and Gambaro, G. (2019) Sodium Fluctuations and Mortality in a General Hospitalized Population; Kidney Blood Press Res. DOI: 10.1159/000500916. 